\title{
Switching Control for Parameter Identifiability of Uncertain Systems
}

\author{
Giorgio Battistelli and Pietro Tesi
}

\begin{abstract}
This paper considers the problem of identifying the parameters of an uncertain linear system by means of feedback control. The problem is approached by considering time-varying controllers. It is shown that even when the uncertainty set is not finite, parameter identifiability can be generically ensured by switching among a finite number of linear time-invariant controllers. The results are shown to have several implications, ranging from fault detection and isolation to adaptive and supervisory control. Practical aspects of the problem are also discussed in details.
\end{abstract}

\section{INTRODUCTION}

Identifying the parameters of an uncertain system from input-output data is a problem of long-standing fundamental interest in control engineering. This problem is often referred to as the problem of parameter identifiability [1], [2]. This paper considers the identifiability problem with respect to uncertain linear systems where the uncertainty set consists of a known bounded set possibly containing a continuum of parameters. For this class of systems, we address the problem of ensuring the identifiability of the unknown parameters of the system by means of feedback controllers.

The motivations for studying this problem are immense. For instance, parameter identifiability of a feedback loop can be of interest in the context of fault detection/isolation for systems subjects to failures, in order to make it possible to promptly detect any departure from the nominal behavior and to precisely identify the parameter variation [3], [4]. Another application is control reconfiguration wherein the objective is that of replacing the active controller (typically designed in order to ensure robust stability in all the uncertainty region) with a different one providing enhanced (possibly optimized) performance [5], [6]. Finally, on-line estimation of the uncertain parameters under feedback can be exploited when dealing with systems which naturally exhibit multiple operating conditions for constructing adaptive control laws. In fact, many existing adaptive control techniques rely on the idea of certainty equivalence, which amounts to applying at each instant of time the controller designed for the model that best fits the available data [7], [8].

In this paper, the problem of parameter identifiability is approached by searching for feedback control laws under which closed-loop behaviors obtained with different system

G. Battistelli is with the University of Florence, Dipartimento di Ingegneria dellInformazione (DINFO), Via di Santa Marta 3, 50139 Firenze, Italy (e-mail: giorgio.battistelli@unifi.it). P. Tesi is with University of Groningen, ENgineering and TEchnology institute Groningen (ENTEG), Faculty of Mathematics and Natural Sciences, Nijenborgh 4, 9747 AG Groningen, The Netherlands (e-mail: p.tesi@rug.nl). parameters can be distinguished one from another. To this end, we introduce a notion of discerning control. Parameter identifiability is then defined precisely in terms of discerning control.

In principle, parameter identifiability under feedback can always be ensured by means of a probing signal injected into the plant as an additive perturbation input, superimposed to the control variable [7], [8]. Nevertheless, in many contexts, such a solution should be avoided due to the inherent drawback of leading the feedback loop away from the desired behavior, thus destroying regulation properties. This is especially true when the behavior of the feedback loop has to be monitored continuously as in the contexts of fault/detection isolation and adaptive control. Then, a natural question arises on whether or not it is possible to guarantee parameter identifiability directly by means of a feedback control law, possibly designed also to satisfy other control objectives (e.g., stability in nominal operating conditions). An affirmative answer to this question was given in [9], [10] for the special case of switching linear systems, i.e., when the uncertain parameters can take on only a finite number of possible values. Specifically, in [9], [10], it is shown that, under quite mild assumptions, for switching linear systems almost all linear time-invariant (LTI) controllers are discerning.

In general, an analogous result cannot be established in case of continuously parameterized systems, i.e., when the uncertainty set is not finite. The reason is inherently tied to the fact that LTI controllers do not generally provide a sufficient level of excitation to the loop [7, Chapter 2]. The problem of loss of identifiability due to feedback can be overcome by means of time-varying controllers, and one possibility is given by switching control [11], [12]. In this paper, we exploit this property to show that parameter identifiability can in fact be generically ensured by switching among a finite number of LTI controllers (hereafter called modes), provided that the number of different controller modes is sufficiently large. Specifically, an upper bound on the number of controller modes needed for parameter identifiability is given in terms of the dimension of the uncertainty set. Moreover, we show that the result remains true even if we restrict the controller modes to be of a given fixed order and to satisfy certain stability requirements. The latter result is perhaps surprising as it indicates that the seemingly conflicting goals of ensuring parameter identifiability as well as a satisfactory behavior of the feedback system (at least under nominal conditions) can be simultaneously accomplished by means of switching control.

As a further contribution, we analyze the properties of 
least-squares parameter estimation in connection with the use of discerning controllers. Specifically, in order to ensure the practical applicability of the estimation technique, we focus on a multi-model approach wherein the estimate is selected among a finite number of possible values of the parameter vector (obtained by suitably sampling the uncertainty set). In this context, a bound on the worst-case parameter estimation error is derived, which accounts also for the presence of unknown but bounded disturbances and measurement noises. This latter result is of special interest in the context of multi-model adaptive switching control (MMASC) of uncertain systems [9], [13]-[16], of which multi-model least-square parameter estimation constitutes one of the key elements. In this respect, it has been shown in [9], [16] that, in the case of a finite uncertainty set, by employing discerning controllers it is possible to construct MMASC schemes which enjoy quite strong stability properties, namely exponential input-to-state stability. Hence, the results of the paper suggest that similar stability properties could be achieved also in the case of continuously parameterized uncertainty. This issue will be the subject of further research.

The remainder of the paper is organized as follows. In Section II, we describe the framework under consideration. In Section III, the main results of the paper are given concerning the existence and genericity of switching controllers ensuring parameter identifiability. Section IV analyzes the properties of multi-model least-squares parameter estimation. Examples are finally given in Section V.

For the sake of clarity, all the proofs are reported in the Appendix section.

Notation. Before concluding this section, let us introduce some notations and basic definitions. Given a vector $v \in \mathbb{R}^{n}$, $|v|$ denotes its Euclidean norm. Given a symmetric, positive semi-definite matrix $P$, we denote by $\lambda_{\min }(P)$ and $\lambda_{\max }(P)$ the minimum and maximum eigenvalues of $P$, respectively. Given a matrix $M, M^{\top}$ is its transpose and $|M|=$ $\left[\lambda_{\max }\left(M^{\top} M\right)\right]^{1 / 2}$ its spectral norm. Given a measurable time function $v: \mathbb{R}^{+} \rightarrow \mathbb{R}^{n}$ and a time interval $\mathcal{I} \subseteq \mathbb{R}^{+}$, we denote the $\mathcal{L}_{2}$ and $\mathcal{L}_{\infty}$ norms of $v(\cdot)$ on $\mathcal{I}$ as $\|v\|_{2, \mathcal{I}}=\sqrt{\int_{\mathcal{I}}|v(t)|^{2} d t}$ and $\|v\|_{\infty, \mathcal{I}}=\operatorname{ess} \sup _{t \in \mathcal{I}}|v(t)|$ respectively. When $\mathcal{I}=R^{+}$, we simply write $\|v\|_{2}$ and $\|v\|_{\infty}$. Finally, we let $\mathcal{L}_{2}(\mathcal{I})$ and $\mathcal{L}_{\infty}(\mathcal{I})$ denote the sets of square integrable and, respectively, (essentially) bounded time functions on $\mathcal{I}$.

\section{FRAMEWORK AND OBJECTIVES}

We consider a process described by an uncertain linear system $\mathcal{P}(\theta)$

$$
\left\{\begin{array}{l}
\dot{x}=A(\theta) x+B(\theta) u \\
y=C(\theta) x
\end{array}\right.
$$

where $x \in \mathbb{R}^{n_{x}}$ is the state, $u \in \mathbb{R}^{n_{u}}$ is the input, $y \in \mathbb{R}^{n_{y}}$ is the output, and $\theta \in \mathbb{R}^{n_{\theta}}$ is an unknown parameter vector belonging to the known bounded set $\Theta \subseteq \mathbb{R}^{n_{\theta}}$.

The problem of interest is that of designing a controller $\mathcal{C}$ ensuring global discernibility, i.e., identifiability of the unknown parameter vector $\theta$ from observations of the plant input/output data

$$
z=\operatorname{col}(u, y) \text {. }
$$

where col stands for column vector. In [10], the problem was addressed in the special case when the set $\Theta$ is finite and it was shown that, under mild assumption, global discernibility can be ensured by means of a LTI controller. When the set $\Theta$ is not finite, a single LTI controller in general cannot ensure global discernibility by itself. Nevertheless, as will be shown in the following, it turns out that global discernibility can be achieved by switching among a finite number of LTI controllers. Accordingly, let the controller be described by a switching linear system

$$
\left\{\begin{array}{l}
\dot{\xi}=F_{\sigma} \xi-G_{\sigma} y \\
u=H_{\sigma} \xi-K_{\sigma} y
\end{array}\right.
$$

where $\xi \in \mathbb{R}^{n_{\xi}}$ is the controller state and $\sigma: \mathbb{R}_{+} \mapsto \mathcal{N}:=$ $\{1,2, \ldots, N\}$ is the switching signal, i.e. the signal (right continuous) which identifies the index of the active system at each instant of time. Hereafter, it will be supposed that the switching signal $\sigma$ is generated so as to have a finite number of discontinuity points in every finite time interval. For any $i \in \mathcal{N}, F_{i}, G_{i}, H_{i}$, and $K_{i}$ are constant matrices of appropriate dimensions. In the sequel, we shall denote by $\mathcal{C}_{i}$ the LTI system with state-space representation $\left\{F_{i}, G_{i}, H_{i}, K_{i}\right\}$ and by $\mathcal{C}_{\sigma}$ the control law associated with the switching signal $\sigma$.

Denote by $\chi=\operatorname{col}(x, \xi)$ and $z=\operatorname{col}(u, y)$ the state and the output of the closed-loop system $\left(\mathcal{P}(\theta) / \mathcal{C}_{\sigma}\right)$ resulting from the interconnection of (1) and (2) when the unknown parameter takes value $\theta$ and the controller switching signal is $\sigma$. The corresponding dynamics can be therefore expressed as

$$
\left\{\begin{array}{l}
\dot{\chi}=\Psi_{\sigma}(\theta) \chi \\
z=\Lambda_{\sigma}(\theta) \chi
\end{array}\right.
$$

where, for any $i \in \mathcal{N}$ and $\theta \in \Theta$,

$$
\begin{aligned}
\Psi_{i}(\theta) & =\left[\begin{array}{cc}
A(\theta)-B(\theta) K_{i} C(\theta) & B(\theta) H_{i} \\
-G_{i} C(\theta) & F_{i}
\end{array}\right], \\
\Lambda_{i}(\theta) & =\left[\begin{array}{cc}
-K_{i} C(\theta) & H_{i} \\
C(\theta) & 0
\end{array}\right] .
\end{aligned}
$$

Finally, let $z\left(t, t_{0}, x_{0}, \xi_{0}, \theta, \sigma\right)$ denote the value at time $t$ of $z$ when the plant initial state at time $t_{0}$ is $x_{0}$, the controller initial state is $\xi_{0}$, the unknown parameter vector takes value $\theta$, and the controller switching signal is $\sigma$. The following notions can be introduced.

Definition 1: Let the process be as in (1) and assume that $u$ and $y$ are available for measurements. Further, consider two distinct parameter vectors $\theta, \theta^{\prime} \in \Theta$. A switching controller of the form (2) is said to be $\left(\theta, \theta^{\prime}\right)$-discerning if, for any timeinterval $\mathcal{I}:=\left[t_{0}, t_{0}+T\right)$, with $T>0$, there exists a switching signal $\sigma: \mathcal{I} \rightarrow \mathcal{N}$ such that

$$
\left\|z\left(\cdot, t_{0}, x_{0}, \xi_{0}, \theta, \sigma\right)-z\left(\cdot, t_{0}, x_{0}^{\prime}, \xi_{0}^{\prime}, \theta^{\prime}, \sigma\right)\right\|_{2, \mathcal{I}} \neq 0
$$

for all nonzero quadruples of vectors $\left(x_{0}, \xi_{0}, x_{0}^{\prime}, \xi_{0}^{\prime}\right)$. In addition, the switching controller (2) is said to be globally 
discerning if it satisfies condition (5) for all pairs $\left(\theta, \theta^{\prime}\right)$ of different parameter vectors.

In words, when the switching controller (2) is $\left(\theta, \theta^{\prime}\right)$ discerning and a discerning switching signal $\sigma$ is adopted, then $\mathcal{P}(\theta)$ and $\mathcal{P}\left(\theta^{\prime}\right)$ cannot give rise to the same observation data when $\mathcal{C}_{\sigma}$ is in the feedback loop (unless the initial conditions are null). As a consequence, under global discernibility, it is possible to uniquely identify the unknown parameter vector $\theta$ by observing $z$ on the interval $\mathcal{I}$.

\section{MAIN RESULTS}

In this section, we derive sufficient conditions for a switching control to be discerning and we show that, under mild assumptions, almost every switching controller is discerning provided that the number $N$ of controller modes is sufficiently large.

To this end, notice preliminarily that a necessary condition for the existence of a discerning controller is that all the pairs $(A(\theta), C(\theta))$ are observable. In fact, the presence of unobservable dynamics would entail the existence of non-zero trajectories of the closed-loop state $\chi$ corresponding to zero trajectories of the closed-loop output $z$ and, hence, for which it would be impossible to infer the plant mode. Accordingly, the following assumption is considered.

A1. The pair $(A(\theta), C(\theta))$ is observable for all $\theta \in \Theta$.

Let now $\varphi_{i}(s, \theta)$ denote the characteristic polynomial of the closed-loop system $\left(\mathcal{P}(\theta) / \mathcal{C}_{i}\right)$ resulting from the feedback interconnection of the plant $\mathcal{P}(\theta)$ with the $i$-th controller mode $\mathcal{C}_{i}$. The following result holds.

Lemma 1: Let assumption A1 hold and suppose that, for any pair of distinct parameter vectors $\theta, \theta^{\prime} \in \Theta$, there exist at least one index $i \in \mathcal{N}$ such that the two closed-loop characteristic polynomials $\varphi_{i}(s, \theta)$ and $\varphi_{i}\left(s, \theta^{\prime}\right)$ are coprime. Then, the following properties are true.

(i) the switching controller (2) is globally discerning;

(ii) condition (5) holds for any switching signal $\sigma$ such that each controller mode $i \in \mathcal{N}$ is active, at least, on an interval $\mathcal{I}_{i} \subset \mathcal{I}$ of positive measure.

Let now $\bar{n}_{\xi}$ denotes the total number of elements of the controller matrices $\left(F_{i}, G_{i}, H_{i}, K_{i}\right)$ when the controller order is $n_{\xi}$, and let $\mathcal{D}\left(\theta, \theta^{\prime}\right) \subseteq \mathbb{R}^{\bar{n}_{\xi}}$ be the set of controller matrices 1 $\left(F_{i}, G_{i}, H_{i}, K_{i}\right)$ for which the two closed-loop polynomials $\varphi_{i}(s, \theta)$ and $\varphi_{i}\left(s, \theta^{\prime}\right)$ are coprime. Further, for a given number $N$ of controller modes, let $\mathcal{D}_{N} \subseteq \mathbb{R}^{N \bar{n}_{\xi}}$ be the set of switching controllers $\left(F_{i}, G_{i}, H_{i}, K_{i}\right), i \in \mathcal{N}$ satisfying the hypothesis of Lemma 1 (i.e., such that for any pair of distinct parameter vectors $\theta, \theta^{\prime} \in \Theta$, there exist at least one index $i \in \mathcal{N}$ such that the two closed-loop characteristic polynomials $\varphi_{i}(s, \theta)$ and $\varphi_{i}\left(s, \theta^{\prime}\right)$ are coprime). Since, in view of Lemma 1, all switching controllers belonging to $\mathcal{D}_{N}$ are globally discerning,

\footnotetext{
${ }^{1}$ Here, with a little abuse of notation, we identify the quadruples $\left(F_{i}, G_{i}, H_{i}, K_{i}\right)$ with an $\bar{n}_{\xi}$-dimensional vector containing all the elements of the matrices $\left(F_{i}, G_{i}, H_{i}, K_{i}\right)$ according to a given order.
}

we are now interested in studying the properties of the sets $\mathcal{D}\left(\theta, \theta^{\prime}\right)$ and $\mathcal{D}_{N}$.

To this end, recall that coprimeness of the two polynomials $\varphi_{i}(s, \theta)$ and $\varphi_{i}\left(s, \theta^{\prime}\right)$ is equivalent to the fact that their Sylvester resultant $R_{i}\left(\theta, \theta^{\prime}\right)$ (i.e., the determinant of the Sylvester matrix associated with the two polynomials) is different from 0 . With this respect, we note that $R_{i}\left(\theta, \theta^{\prime}\right)$ depends polynomially on the elements of the controller matrices $\left(F_{i}, G_{i}, H_{i}, K_{i}\right)$. Hence, when only a single pair of distinct parameter vectors $\theta, \theta^{\prime} \in \Theta$ is taken into account, the set of controller matrices $\left(F_{i}, G_{i}, H_{i}, K_{i}\right)$ for which $R_{i}\left(\theta, \theta^{\prime}\right)=0$, which is the complement of $\mathcal{D}\left(\theta, \theta^{\prime}\right)$, is an algebraic set. Then, as well known, only two situations may occur: either $R_{i}\left(\theta, \theta^{\prime}\right)=0$ is always satisfied; or $R_{i}\left(\theta, \theta^{\prime}\right)=0$ is satisfied on a set with zero Lebesgue measure. In this latter case, the set $\mathcal{D}\left(\theta, \theta^{\prime}\right)$ is generic 2 (since it is the complement of a proper algebraic set). The following lemma, proved in [9], provides sufficient conditions for such a favorable situation to occur.

Lemma 2: [9] Let assumption A1 hold and consider two distinct parameter vectors $\theta, \theta^{\prime} \in \Theta$. Then a controller $\left(F_{i}, G_{i}, H_{i}, K_{i}\right)$ ensuring coprimeness of the two polynomials $\varphi_{i}(s, \theta)$ and $\varphi_{i}\left(s, \theta^{\prime}\right)$ exists if and only if the following two conditions hold:

(a) the transfer functions of $\mathcal{P}(\theta)$ and $\mathcal{P}\left(\theta^{\prime}\right)$ are different;

(b) the characteristic polynomials of the uncontrollable parts of $\mathcal{P}(\theta)$ and $\mathcal{P}\left(\theta^{\prime}\right)$ are coprime.

In addition, when conditions (a)-(b) holds, for any given controller order $n_{\xi}$ the set $\mathcal{D}\left(\theta, \theta^{\prime}\right)$ is generic and of full measure on $\mathbb{R}^{\bar{n}_{\xi}}$.

Building on the above lemmas, under suitable regularity assumptions for the functions $A(\theta), B(\theta), C(\theta)$, it is possible to derive conditions for the existence of a global discerning switching controller on the whole uncertainty set $\Theta$. In particular, by exploiting the results of [17], the following theorem can be stated.

Theorem 1: Let the uncertainty set $\Theta$ be contained in an analytic manifold $\mathcal{M} \subset \mathbb{R}^{n_{\theta}}$ of dimension $M$ and let the elements of the system matrices $A(\theta), B(\theta), C(\theta)$ be analytic functions of $\theta$ on $\mathcal{M}$. Further, let assumption A1 and conditions (a)-(b) of Lemma 2 hold on $\mathcal{M}$. Then, provided that $N \geq 2 M+1$, the set $\mathcal{D}_{N}$ is generic and of full measure on $\mathbb{R}^{N \bar{n}_{\xi}}$

A few remarks are in order. First of all, notice that Theorem 1 provides a bound on the number $N$ of controller modes that may be needed in order to ensure identifiability of the unknown parameter $\theta$ in $\Theta$. Such a bound is consistent with the results of [9], [10] where it is shown that when the set $\Theta$ is finite, i.e., it is a 0 -dimensional manifold, one single LTI controller is generically discerning. As discussed in [17], the bound $N \geq 2 M+1$ for parameter distinguishability

\footnotetext{
${ }^{2}$ Recall that a subset $\mathcal{X}$ of a topological space is generic when it is open and dense: for any $x \in \mathcal{X}$, then there exists a neighborhood of $x$ contained in $\mathcal{X}$; for any $x \notin \mathcal{X}$, then every neighborhood of $x$ contains an element of $\mathcal{X}$.
} 
is tight for general maps (in the sense that when $N<$ $2 M+1$ one can find counterexamples). However, for specific cases, fewer controller modes can be sufficient. For instance, when $\theta$ is a scalar parameter, one can consider a single LTI controller $\left(F_{i}, G_{i}, H_{i}, K_{i}\right)$ and plot the root locus of the closed loop polynomial $\varphi_{i}(s, \theta)$ as a function of $\theta$. Then, global discernibility is guaranteed provided that such a root locus never cross itself.

Notice finally that the set of analytic functions considered in the statement of Theorem 1 is quite general as it captures many function classes of interest (e.g., polynomials, trigonometric functions, exponentials, and also rational functions as long as away from singularities).

\section{A. Accounting for additional control objectives}

In the foregoing analysis, only the global discernibility objective has been taken into account. However generally speaking, a control law should typically satisfy other control objectives, the most fundamental one being stability. With this respect, suppose that we want the switching controller to ensure closed-loop stability in a given subset $\bar{\Theta}$ of $\Theta$ together with global discernibility. For instance, $\bar{\Theta}$ can represent the neighborhood of the nominal operating condition and the switching controller should be designed so as to ensure: a satisfactory behavior in nominal conditions as well as the possibility of promptly identifying any departure from the nominal behavior (e.g, for fault-detection and isolation or for control reconfiguration purposes). An extreme case is when $\bar{\Theta}=\Theta$ so that we want to design a robust and globally discerning switching controller ensuring stability for any possible operating condition (of course this may be possible or not depending on the of size of the uncertainty set $\Theta$ ).

As well known, a sufficient condition to ensure stability under switching is the existence of a common Lyapunov function. For example, if we consider a quadratic parameterdependent Lyapunov function $v(\chi)=\chi^{\top} \Pi(\theta) \chi$, then in order for the closed-loop system $\left(\mathcal{P}(\theta), \mathcal{C}_{\sigma}\right)$ to be stable for any $\theta \in \bar{\Theta}$ it is sufficient that there exists $\Pi(\theta)=\Pi(\theta)^{\top}$ such that

$$
\begin{aligned}
& \Pi(\theta) \succ 0, \\
& \Psi_{i}(\theta)^{\top} \Pi(\theta)+\Pi(\theta) \Psi_{i}(\theta) \prec 0,
\end{aligned}
$$

for any $i \in \mathcal{N}$ and for any $\theta \in \bar{\Theta}$. When the set $\Theta$ is compact, by means of simple continuity arguments, it is immediate to show that, for any given smooth $\Pi(\theta)$, the set of controller matrices $\left(F_{i}, G_{i}, H_{i}, K_{i}\right)$ satisfying (7) is an open subset of $\mathbb{R}^{\bar{n}_{\xi}}$. Then, recalling that, by definition, any non-empty open set contains a closed-ball of positive radius, the following result on the existence of global discerning controllers ensuring also stability can be readily stated.

Theorem 2: Let the same hypotheses of Theorem 11 hold. Further, let the set $\bar{\Theta}$ be compact and suppose that there exists at least one controller $\left(F_{i}, G_{i}, H_{i}, K_{i}\right)$ for which conditions (6) and (7) are satisfied with the Lyapunov matrix $\Pi(\theta)$ depending continuously on $\theta$. Then, whenever $N \geq 2 M+1$, the set of switching controllers $\left(F_{i}, G_{i}, H_{i}, K_{i}\right), i \in \mathcal{N}$ that jointly satisfies (6) and (7) and ensures global discernibility is non-negligible, in the sense that it contains a ball of positive radius in $\mathbb{R}^{N \bar{n}_{\xi}}$.

\section{Multi-MOdel LEAST-SQUARES PARAMETER ESTIMATION}

In this section, we discuss how the unknown parameter vector $\theta$ can be estimated from the closed-loop data $z$ on an interval $\mathcal{I}=\left[t_{0}, t_{0}+T\right]$ and we show that, when the data result from application of a discerning switching controller, the resulting estimate enjoys some nice properties even in the presence of unknown disturbances and measurement noises.

To this end, recall that, when the unknown parameter vector takes value $\theta$, the evolution of $z$ on the interval $\mathcal{I}$ takes the form $z\left(t, t_{0}, x_{0}, \xi_{0}, \theta, \sigma\right)$. Then the set $\mathcal{S}_{\sigma}(\theta)$ of all possible closed-loop data on the interval $\mathcal{I}$ associated with $\theta$ and with the switching signal $\sigma$ can be written as

$$
\begin{gathered}
\mathcal{S}_{\sigma}(\theta)=\left\{\hat{z} \in \mathcal{L}_{2}(\mathcal{I}): \hat{z}(\cdot)=z\left(\cdot, t_{0}, \hat{x}_{0}, \hat{\xi}_{0}, \theta, \sigma\right) \text { on } \mathcal{I}\right. \\
\text { for some } \left.\hat{x}_{0} \in \mathbb{R}^{n_{x}}, \hat{\xi}_{0} \in \mathbb{R}^{n_{\xi}}\right\} .
\end{gathered}
$$

Hence a natural approach for estimating the plant unknown parameters is the least-squares one, which amounts to selecting the parameter vector $\theta$ for which the distance between the observed close-loop data $z$ on the interval $\mathcal{I}$ and the set $\mathcal{S}_{\sigma}(\theta)$ is minimal. Accordingly, the optimal least-squares estimate $\hat{\theta}^{\circ}$ can be obtained as

$$
\hat{\theta}^{\circ} \in \arg \min _{\hat{\theta} \in \Theta} \delta_{\sigma}(z, \hat{\theta})
$$

where

$$
\delta_{\sigma}(z, \hat{\theta})=\min _{\hat{x}_{0} \in \mathbb{R}^{n_{x}}, \hat{\xi}_{0} \in \mathbb{R}^{n_{\xi}}}\left\|z(\cdot)-z\left(\cdot, t_{0}, \hat{x}_{0}, \hat{\xi}_{0}, \hat{\theta}, \sigma\right)\right\|_{2, \mathcal{I}} .
$$

Concerning the computation of the distance (9), for any possible feedback loop $\left(\mathcal{P}(\theta), \mathcal{C}_{\sigma}\right)$, let $\Phi_{\sigma}\left(t, t_{0}, \theta\right)$ denote its state transition matrix and let $W_{\sigma}(\theta)$ be its observability Gramian on the interval $\mathcal{I}$, i.e.,

$$
W_{\sigma}(\theta)=\int_{\mathcal{I}} \Phi_{\sigma}\left(t, t_{0}, \theta\right)^{\top} \Lambda_{\sigma(t)}(\theta)^{\top} \Lambda_{\sigma(t)}(\theta) \Phi_{\sigma}\left(t, t_{0}, \theta\right) d t .
$$

Notice that, for any globally discerning switching control law, the observability Gramian $W_{\sigma}(\theta)$ turns out to be positive definite for any $\theta \in \Theta$ (otherwise there would be zero output trajectories corresponding to non-zero state trajectories and parameter identification would not be possible). Hence, in this case, the minimization in (9) yields

$$
\begin{gathered}
\delta_{\sigma}(z, \hat{\theta})=\left(\int_{\mathcal{I}} \mid z(t)-\Lambda_{\sigma(t)}(\hat{\theta}) \Phi_{\sigma}\left(t, t_{0}, \hat{\theta}\right)\left(W_{\sigma}(\hat{\theta})\right)^{-1}\right. \\
\left.\times\left.\int_{\mathcal{I}} \Phi_{\sigma}\left(\tau, t_{0}, \hat{\theta}\right)^{\top} \Lambda_{\sigma(\tau)}(\hat{\theta})^{\top} z(\tau) d \tau\right|^{2} d t\right)^{1 / 2} .
\end{gathered}
$$

For further considerations on how this quantity can be computed in practice the interested reader is referred to Appendix A of [16], where a similar problem is addressed.

From Definition 1, it is immediately clear that when a globally discerning switching control law is adopted, for any 
non null $z$ the $\delta_{\sigma}(z, \hat{\theta})$ is zero if and only if $\hat{\theta}$ coincides with $\theta$, the true parameter vector.

Proposition 1: Let the switching controller (2) be globally discerning and a discerning switching signal $\sigma$ be adopted on the observation interval $\mathcal{I}$. Further, let the observed data $z$ be generated by the closed-loop system (3) from initial condition $\chi_{0}=\left(x_{0}, \xi_{0}\right) \neq 0$. Then, $\hat{\theta}^{\circ}=\theta$.

While the above proposition illustrates the theoretical effectiveness of the leas-squares estimation criterion in ideal conditions, in practice computation of the minimum in (8) can be a quite challenging task when the set $\Theta$ is not finite. In this case, a standard approach is the multi-model one which amounts to considering only a finite number, say L, of possible parameter values by constructing the finite set $\Theta_{L}=\left\{\theta_{\ell}, \ell=1, \ldots, L\right\} \subseteq \Theta$. Typically, $\Theta_{L}$ is obtained by sampling the set $\Theta$ with a given guaranteed density $\varepsilon$, so that for any $\theta \in \Theta$ there exists at least one $\theta_{\ell} \in \Theta_{L}$ such that $\left|\theta-\theta_{\ell}\right| \leq \epsilon$. When such a condition is satisfied, we say that $\Theta_{L}$ is $\epsilon$-dense in $\Theta$. Accordingly, the following multi-model least squares criterion can be used to estimate the unknown parameter vector $\theta$

$$
\hat{\theta}_{L} \in \arg \min _{\hat{\theta} \in \Theta_{L}} \delta_{\sigma}(z, \hat{\theta})
$$

as an alternative to (8).

Remark 1: Guidelines on how to choose an $\epsilon$-dense finite covering for $\Theta$ can be found, for instance, in [18]-[20].

\section{A. Properties of multi-model least-squares parameter estima- tion}

When analyzing of the properties of an estimation criterion, either optimal as in (8) or approximate as in (10), it is important to take into account also the effects of process disturbances and measurement noises. With this respect, in the following analysis we suppose that the plant state and measurement equations are affected by additive disturbances $d$ and $n$, respectively, i.e.,

$$
\mathcal{P}(\theta):\left\{\begin{array}{l}
\dot{x}=A(\theta) x+B(\theta) u+d \\
y=C(\theta) x+n
\end{array}\right.
$$

with $d \in \mathbb{R}^{n_{x}}$ and $n \in \mathbb{R}^{n_{y}}$. Then, it is an easy matter to verify that a state space representation of the closed-loop system $\left(\mathcal{P}(\theta) / \mathcal{C}_{\sigma}\right)$ takes the form

$$
\left\{\begin{aligned}
\dot{\chi} & =\Psi_{\sigma}(\theta) \chi+\Xi_{\sigma}(\theta) v \\
z & =\Lambda_{\sigma}(\theta) \chi+\Gamma_{\sigma} v
\end{aligned}\right.
$$

where $v=(d, n)$ and

$$
\Xi_{i}(\theta)=\left[\begin{array}{cc}
I & B(\theta) K_{i} \\
0 & G_{i}
\end{array}\right], \quad \Gamma_{i}=\left[\begin{array}{cc}
0 & K_{i} \\
0 & I
\end{array}\right],
$$

for any $i \in \mathcal{N}$ and $\theta \in \Theta$. Further, thanks to linearity, the closed-loop data $z$ can be decomposed as

$$
z=z^{(\mathrm{n})}+z^{(\mathrm{f})}
$$

where $z^{(\mathrm{f})}$ is the forced response and $z^{(\mathrm{n})}(t)$ is the natural response which can be written as

$$
z^{(\mathrm{n})}(t)=z\left(t, t_{0}, x_{0}, \xi_{0}, \theta, \sigma\right)
$$

with $z\left(t, t_{0}, x_{0}, \xi_{0}, \theta, \sigma\right)$ the same function of the previous sections. Notice also that the forced response $z^{(\mathrm{f})}$ can be bounded in terms of the disturbance amplitude as follows.

Proposition 2: Let the set $\Theta$ be compact and let the elements of $A(\theta), B(\theta)$, and $C(\theta)$ depend continuously on $\theta$. Then, for any $\mathcal{I}=\left[t_{0}, t_{0}+T\right]$, there exists a positive real $\gamma$ such that

$$
\left\|z^{(\mathrm{f})}\right\|_{2, \mathcal{I}} \leq \gamma\|v\|_{\infty, \mathcal{I}}
$$

Notice now that, by virtue of the triangular inequality, we have

$$
\delta_{\sigma}\left(z^{(\mathrm{n})}, \hat{\theta}\right)-\left\|z^{(\mathrm{f})}\right\|_{2, \mathcal{I}} \leq \delta_{\sigma}(z, \hat{\theta}) \leq \delta_{\sigma}\left(z^{(\mathrm{n})}, \hat{\theta}\right)+\left\|z^{(\mathrm{f})}\right\|_{2, \mathcal{I}}
$$

for any $\hat{\theta} \in \Theta$. Hence, the properties of the least-squares estimate $\hat{\theta}_{L}$ can be investigated by deriving bounds on $\delta_{\sigma}\left(z^{(\mathrm{n})}, \hat{\theta}\right)$. In this respect, the following result is relevant.

Proposition 3: Consider the same assumptions as in Proposition 1 Then, for any $\hat{\theta} \in \Theta$,

$$
\begin{aligned}
& {\left[\delta_{\sigma}\left(z^{(\mathrm{n})}, \hat{\theta}\right)\right]^{2}} \\
& \quad=\left[\begin{array}{r}
\chi_{0} \\
-V_{\sigma}(\theta, \hat{\theta}) \chi_{0}
\end{array}\right]^{\top} W_{\sigma}(\theta, \hat{\theta})\left[\begin{array}{r}
\chi_{0} \\
-V_{\sigma}(\theta, \hat{\theta}) \chi_{0}
\end{array}\right]
\end{aligned}
$$

where

$$
\begin{aligned}
U_{\sigma}(\theta, \hat{\theta})= & \int_{\mathcal{I}} \Phi_{\sigma}\left(\tau, t_{0}, \hat{\theta}\right)^{\top} \Lambda_{\sigma(\tau)}(\hat{\theta})^{\top} \\
& \times \Lambda_{\sigma(\tau)}(\theta) \Phi_{\sigma}\left(\tau, t_{0}, \theta\right) d \tau \\
V_{\sigma}(\theta, \hat{\theta})= & \left(W_{\sigma}(\hat{\theta})\right)^{-1} U_{\sigma}(\theta, \hat{\theta}) \\
W_{\sigma}(\theta, \hat{\theta})= & {\left[\begin{array}{ll}
W_{\sigma}(\theta) & U_{\sigma}(\theta, \hat{\theta})^{\top} \\
U_{\sigma}(\theta, \hat{\theta}) & W_{\sigma}(\hat{\theta})
\end{array}\right] . }
\end{aligned}
$$

For any pair of parameter vectors $\theta, \hat{\theta} \in \Theta$, the joint observability Gramian $W_{\sigma}(\theta, \hat{\theta})$ provides information concerning the degree of distinguishability between the two closed-loop systems $\left(\mathcal{P}(\theta), \mathcal{C}_{\sigma}\right)$ and $\left(\mathcal{P}(\hat{\theta}), \mathcal{C}_{\sigma}\right)$. In fact, whenever the switching control law $\mathcal{C}_{\sigma}$ is globally discerning, the matrix $W_{\sigma}(\theta, \hat{\theta})$ is singular if and only if $\theta=\hat{\theta}$ (this is a straightforward consequence of Proposition 11). Then, we can derive the following result.

Lemma 3: Let the same assumptions as in Proposition 1 hold. Further, let the set $\Theta$ be compact and let the elements of $A(\theta), B(\theta)$, and $C(\theta)$ depend continuously on $\theta$. Moreover, let the switching controller (2) be globally discerning and a discerning switching signal $\sigma$ be adopted on the observation interval $\mathcal{I}$. Then, there exist two class $\mathcal{K}$ function $\sqrt{3} \alpha(\cdot)$ and $\beta(\cdot)$ such that

$$
\alpha(|\theta-\hat{\theta}|)\left|\chi_{0}\right| \leq \delta_{\sigma}\left(z^{(\mathrm{n})}, \hat{\theta}\right) \leq \beta(|\theta-\hat{\theta}|)\left|\chi_{0}\right|
$$

${ }^{3}$ Recall that a function $\varphi: \mathbb{R}^{+} \rightarrow \mathbb{R}^{+}$belongs to class $\mathcal{K}$ if it is continuous, strictly increasing, and $\varphi(0)=0$. 
for any $\hat{\theta} \in \Theta$.

The importance of Lemma 3 is that it allows to bound the distance $\delta_{\sigma}\left(z^{(\mathrm{n})}, \hat{\theta}\right)$, pertaining to the noise-free dynamics, in terms of the initial state $\chi_{0}$ and of the distance between the true parameter vector $\theta$ and the candidate estimate $\hat{\theta}$. In particular, the left-most inequality in (19) ensures that $\delta_{\sigma}\left(z^{(\mathrm{n})}, \hat{\theta}\right)$ cannot be small when the discrepancy $\theta-\hat{\theta}$ is large, whereas the right-most inequality ensures that $\delta_{\sigma}\left(z^{(\mathrm{n})}, \hat{\theta}\right)$ nicely degrades to 0 as the estimate $\hat{\theta}$ approaches the true value $\theta$. With respect to the latter observation, from (19) it follows that when $\Theta_{L}$ is $\epsilon$-dense in $\Theta$ there always exists a parameter vector $\theta_{\ell} \in \Theta_{L}$ such that $\delta_{\sigma}\left(z^{(\mathrm{n})}, \theta_{\ell}\right) \leq \beta(\epsilon)\left|\chi_{0}\right|$. By exploiting inequalities (15) and (19), the main result of this section can finally be stated.

Theorem 3: Let the same assumptions as in Lemmaß hold. Further, let $\Theta_{L}$ be $\epsilon$-dense in $\Theta$. Then, the estimate $\hat{\theta}_{L}$ obtained as in (10) is such that

$$
\left|\theta-\hat{\theta}_{L}\right| \leq \alpha^{-1}\left(\beta(\varepsilon)+\frac{2 \gamma\|v\|_{\infty, \mathcal{I}}}{\left|\chi_{0}\right|}\right)
$$

where $\alpha^{-1}(\cdot)$ is the inverse of $\alpha(\cdot)$.

Concerning the upper bound on the estimation error provided by inequality (20), it can be seen that the term $\beta(\varepsilon)$ (which decreases the denser the sampling $\Theta_{L}$ is) accounts for the fact that only a finite number of models is considered, while the term $2 \gamma\|v\|_{\infty, \mathcal{I}} /\left|\chi_{0}\right|$ can be seen as a sort of noiseto-signal ratio, and indeed goes to 0 as the disturbance $\|v\|_{\infty, \mathcal{I}}$ goes to 0 .

\section{AN EXAMPLE}

In the following, a simple example is provided in order to illustrate how identifiability of an uncertain parameter vector can be achieved my means of switching control. To this end, consider an LTI plant with system matrices

$$
A(\theta)=\left[\begin{array}{rr}
0 & 1 \\
0 & -a
\end{array}\right], \quad B(\theta)=\left[\begin{array}{l}
0 \\
b
\end{array}\right], \quad C(\theta)=\left[\begin{array}{ll}
1 & 0
\end{array}\right],
$$

with $\theta=(a, b)$, and let the switching controller be a purely proportional one

$$
u=-K_{\sigma} y .
$$

Since, the plant transfer matrix is $P(s, \theta)=b /[s(s+a)]$, each closed-loop characteristic polynomial takes the form $\varphi_{i}(s, \theta)=s^{2}+a s+b K_{i}$.

\section{A. One uncertain parameter}

Suppose first, for illustration purpose, that only the gain $b$ is uncertain whereas $a$ is perfectly known, i.e., $\Theta=\left\{(a, b): a=a_{0}, b \in\left[b_{1}, b_{2}\right]\right\}$. Notice that assumption A1 holds whenever $a_{0} \neq 0$. Hence, in this case, we can exploit Lemma 1 and consider, for any pair $b, b^{\prime}$ the two polynomials

$$
\begin{aligned}
& \varphi_{i}(s, \theta)=s^{2}+a_{0} s+b K_{i}, \\
& \varphi_{i}\left(s, \theta^{\prime}\right)=s^{2}+a_{0} s+b^{\prime} K_{i} .
\end{aligned}
$$

As it can be easily verified, the Sylvester resultant of such polynomials is

$$
R_{i}\left(\theta, \theta^{\prime}\right)=K_{i}^{2}\left(b-b^{\prime}\right)^{2} .
$$

Then, it can be seen that if $K_{i} \neq 0$, the resultant is different from 0 whenever $b$ and $b^{\prime}$ are different. Hence, in this case, a single proportional controller with non-null gain is globally discerning and there is no need for considering a switching controller in that $\mathcal{D}_{1}=\left\{K_{1} \neq 0\right\}$. Similar considerations hold when $a$ is uncertain and $b$ is perfectly known.

\section{B. Two uncertain parameters}

Suppose now that both $a$ and $b$ are uncertain, i.e., $\Theta=\left\{(a, b): a \in\left[a_{1}, a_{2}\right], b \in\left[b_{1}, b_{2}\right]\right\}$. Again, assumption A1 holds provided that $0 \notin\left[a_{1}, a_{2}\right]$. Straightforward calculations allow to see that, in this case, the resultant of the two polynomials

$$
\begin{aligned}
& \varphi_{i}(s, \theta)=s^{2}+a s+b K_{i}, \\
& \varphi_{i}\left(s, \theta^{\prime}\right)=s^{2}+a^{\prime} s+b^{\prime} K_{i}
\end{aligned}
$$

is

$$
R_{i}\left(\theta, \theta^{\prime}\right)=K_{i}^{2}\left(b-b^{\prime}\right)^{2}-K_{i}\left(b a^{\prime}-a b^{\prime}\right)\left(a-a^{\prime}\right) .
$$

Hence, a single controller is not sufficient for global discernibility as by choosing

$$
\left(b-b^{\prime}\right)^{2}=\varepsilon, \quad=K_{i} \varepsilon
$$

one has $R_{i}\left(\theta, \theta^{\prime}\right)=0$. In fact, since $\varepsilon$ can be arbitrarily small, it is always possible to find $a, b, a^{\prime}, b^{\prime}$ so as to satisfy 210 regardless of the amplitude of the uncertainty set $\Theta$. Then $\mathcal{D}_{1}=\emptyset$.

On the contrary, it can be seen that a switching controller with two modes, $N=2$, is generically globally discerning. To see this, notice that

$$
\left[\begin{array}{l}
R_{1}\left(\theta, \theta^{\prime}\right) \\
R_{2}\left(\theta, \theta^{\prime}\right)
\end{array}\right]=\left[\begin{array}{ll}
K_{1}^{2} & -K_{1} \\
K_{2}^{2} & -K_{2}
\end{array}\right]\left[\begin{array}{l}
\left(b-b^{\prime}\right)^{2} \\
\left(b a^{\prime}-a b^{\prime}\right)\left(a-a^{\prime}\right)
\end{array}\right]
$$

and

$$
\operatorname{det}\left[\begin{array}{ll}
K_{1}^{2} & -K_{1} \\
K_{2}^{2} & -K_{2}
\end{array}\right]=K_{1} K_{2}\left(K_{2}-K_{1}\right) \text {. }
$$

If we choose $K_{1}$ and $K_{2}$ such that $K_{1} \neq K_{2}, K_{1} \neq 0$, and $K_{2} \neq 0$ the above determinant turns out to be different from 0 . As a consequence, in this case, the two resultants $R_{1}\left(\theta, \theta^{\prime}\right)$ and $R_{2}\left(\theta, \theta^{\prime}\right)$ can simultaneously vanish if and only if $\left(b-b^{\prime}\right)^{2}=0$ and $\left(b a^{\prime}-a b^{\prime}\right)\left(a-a^{\prime}\right)=0$ which is equivalent to $a=a^{\prime}$ and $b=b^{\prime}$. Hence, we have that $\mathcal{D}_{2}=\left\{\left(K_{1}, K_{2}\right): K_{1} \neq\right.$ $\left.K_{2}, K_{1} \neq 0, K_{2} \neq 0\right\}$ which is generic and of full measure in $\mathbb{R}^{2}$.

\section{CONCLUSions}

In this paper, we have addressed the problem of identifying the parameters of an uncertain linear system by means of switching control. It was shown that even when the uncertainty set is not finite, parameter identifiability can be generically ensured by switching among a finite number of linear timeinvariant controllers. In particular, the results show that an 
upper bound on the number of controller modes needed for parameter identifiability can be given in terms of the dimension of the uncertainty set. The results also indicate that the seemingly conflicting goals of ensuring parameter identifiability as well as a satisfactory behavior of the feedback system can be simultaneously accomplished by means of switching control.

Several practical aspects have also been discussed. In particular, we have analyzed the properties of least-squares parameter estimation in connection with the use of discerning controllers, providing bounds on the worst-case parameter estimation error in the presence of: i) finite covering of the uncertainty set; and ii) bounded disturbances affecting the process dynamics as well as measurement noises.

The results lend themselves to be extended in various directions. Most notably, these results find a very natural application in the context of switching control for uncertain systems. In this respect, we envision that the analysis tools introduced in this paper should lead to the development of novel control reconfiguration algorithms capable of achieving input-to-state stability for uncertain systems even when the uncertainty set is described by a continuum.

\section{APPENDIX}

Proof of Lemma 1: Consider two distinct parameter vectors $\theta, \theta^{\prime} \in \Theta$ and consider an index $i$ for which $\varphi_{i}(s, \theta)$ and $\varphi_{i}\left(s, \theta^{\prime}\right)$ are coprime. Let the controller $\mathcal{C}_{i}$ be active on an interval $\mathcal{I}_{i}=[\underline{t}, \bar{t}] \subset \mathcal{I}$. Consider now a nonzero quadruples of vectors $\left(x_{0}, \xi_{0}, x_{0}^{\prime}, \xi_{0}^{\prime}\right)$ representing possible initial states of the two feedback loops $\left(\mathcal{P}(\theta) / \mathcal{C}_{\sigma}\right)$ and $\left(\mathcal{P}\left(\theta^{\prime}\right) / \mathcal{C}_{\sigma}\right)$ at time $t_{0}$. Let $\left(\underline{x}, \underline{\xi}, \underline{x}^{\prime}, \underline{\xi}^{\prime}\right)$ be the corresponding states that are reached at time $\underline{\underline{t}}$, i.e., at the beginning of the time interval $\mathcal{I}_{i}$, under the switching law $\sigma$. Suppose now the switching signal $\sigma$ is chosen so as to satisfy a dwell-time condition, i.e., in such a way that there exists a lower bound $\tau_{\text {dwell }}$ on the time interval between subsequent variations of the controller index. Then, it is immediate to see that, under such a switching law, when $\left(x_{0}, \xi_{0}, x_{0}^{\prime}, \xi_{0}^{\prime}\right) \neq 0$ then also $\left(\underline{x}, \underline{\xi}, \underline{x}^{\prime}, \underline{\xi}^{\prime}\right) \neq 0$. In fact, such a state is reached after switching a finite number of times between autonomous linear systems, i.e., the feedback loops, and it is known that an autonomous linear system cannot reach the zero state in finite time starting from a non-zero initial state. Notice now that, under assumption A1, coprimeness of the polynomials $\varphi_{i}(s, \theta)$ and $\varphi_{i}\left(s, \theta^{\prime}\right)$ implies observability of the parallel system

$$
\left\{\begin{array}{l}
{\left[\begin{array}{c}
\dot{\chi} \\
\dot{\chi}^{\prime}
\end{array}\right]=\left[\begin{array}{cc}
\Psi_{i}(\theta) & 0 \\
0 & \Psi_{i}(\theta)
\end{array}\right]\left[\begin{array}{c}
\chi \\
\chi^{\prime}
\end{array}\right]} \\
\tilde{z}=\left[\Lambda_{i}(\theta)-\Lambda_{i}(\theta)\right]\left[\begin{array}{c}
\chi \\
\chi^{\prime}
\end{array}\right]
\end{array}\right.
$$

(see for instance Proposition 1 of [9]). Then, if we initialize such a system as $\left(\chi(\underline{t}), \chi^{\prime}(\underline{t})\right)=\left(\underline{x}, \underline{\xi}, \underline{x}^{\prime}, \underline{\xi}^{\prime}\right) \neq 0$ at time $\underline{t}$, we have that $\tilde{z}$ is different from 0 a.e. on $\mathcal{I}_{i}=[\underline{t}, \bar{t}]$, where "a.e." stands for "almost everywhere", i.e. everywhere except on a set of zero Lebesgue measure. This, in turn, implies that $z(t, \underline{t}, \underline{x}, \underline{\xi}, \theta, i) \neq z\left(t, \underline{t}, \underline{x}^{\prime}, \underline{\xi}^{\prime}, \theta^{\prime}, i\right)$, or equivalently, $z\left(t, t_{0}, x_{0}, \xi_{0}, \theta, \sigma\right) \neq z\left(t, t_{0}, x_{0}^{\prime}, \xi_{0}^{\prime}, \bar{\theta}^{\prime}, i\right)$, a.e. on $\mathcal{I}_{i}=[\underline{t}, \bar{t}]$.
Then, by choosing a switching signal $\sigma$ which satisfies a dwelltime condition and is such that each controller mode $i$ is active, at least, on an interval $\mathcal{I}_{i} \subset \mathcal{I}$ of positive measure, the same line reasoning can be repeated for any pair $\theta, \theta^{\prime} \in \Theta$ with $\theta \neq \theta^{\prime}$, thus concluding the proof.

Proof of Theorem 1: Notice first that the resultant $R_{i}\left(\theta, \theta^{\prime}\right)$ of the two polynomials $\varphi_{i}(\theta)$ and $\varphi_{i}\left(\theta^{\prime}\right)$ is a polynomial (and hence analytic) function of the elements of the matrices $(A(\theta), B(\theta), C(\theta)),\left(A\left(\theta^{\prime}\right), B\left(\theta^{\prime}\right), C\left(\theta^{\prime}\right)\right)$, and $\left(F_{i}, G_{i}, H_{i}, K_{i}\right)$. This, in turn, implies that $R_{i}\left(\theta, \theta^{\prime}\right)$ is an analytic function of $\theta$ and $\theta^{\prime}$ (since the composition of analytic functions is analytic). Notice now that, under the stated hypotheses, Lemma 2 ensures that, for any pair $\theta, \theta^{\prime} \in \mathcal{M}$ with $\theta \neq \theta^{\prime}$, it is possible to find at least one set of matrices $\left(F_{i}, G_{i}, H_{i}, K_{i}\right)$ such that $\varphi_{i}(\theta)$ and $\varphi_{i}\left(\theta^{\prime}\right)$ are coprime and, hence, $R_{i}\left(\theta, \theta^{\prime}\right) \neq 0$. Recall, finally, that the set $\mathcal{D}_{N}$ corresponds to the set of switching controllers $\left(F_{i}, G_{i}, H_{i}, K_{i}\right) i \in$ $\mathcal{N}$ for which the vector function $\operatorname{col}\left(R_{i}\left(\theta, \theta^{\prime}\right), i \in \mathcal{N}\right)$ is different from 0 for any pair $\theta, \theta^{\prime} \in \mathcal{M}$ with $\theta \neq \theta^{\prime}$. Then, proceeding as in the proof of Theorem 2 of [17], we can conclude that $\mathcal{D}_{N}$ is generic and of full measure on $\mathbb{R}^{N \bar{n}_{\xi}}$ whenever $N \geq 2 M+1$.

Proof of Theorem 2: Let $\left(F_{i}, G_{i}, H_{i}, K_{i}\right)$ be a controller for which conditions (6) and (7) are satisfied with $\Pi(\theta)$ continuous in $\theta$. Further, consider a closed ball $\mathcal{B}(\varepsilon)$ in the controller parameter space $\mathbb{R}^{\bar{n}_{\xi}}$ centered in $\left(F_{i}, G_{i}, H_{i}, K_{i}\right)$ and with radius $\varepsilon$ and let

$$
\begin{aligned}
& \beta(\varepsilon)= \\
& \max _{(F, G, H, K) \in \mathcal{B}(\varepsilon)} \max _{\theta \in \bar{\Theta}} \lambda_{\max }\left\{\Psi_{i}(\theta)^{\top} \Pi(\theta)+\Pi(\theta) \Psi_{i}(\theta)\right\} .
\end{aligned}
$$

Note that, in view of the compactness of $\bar{\Theta}$ and of the continuity of $\Psi(\theta)$ and $\Pi(\theta)$, we have that $\max _{\theta \in \bar{\Theta}} \lambda_{\max }\left\{\Psi_{i}(\theta)^{\top} \Pi(\theta)+\Pi(\theta) \Psi_{i}(\theta)\right\}=\beta(0)<0$. Moreover, under the considered hypotheses, it is easy to show that $\beta(\varepsilon)$ depends continuously on $\varepsilon$ (in this respect, notice that $\Psi_{i}(\theta)$ is an affine function of the controller matrices $\left.\left(F_{i}, G_{i}, H_{i}, K_{i}\right)\right)$. Hence, this implies the existence of $\varepsilon>0$ such that $\beta(\varepsilon)<0$, i.e., such that all the controllers in $\mathcal{B}(\varepsilon)$ satisfies (7) with the same Lyapunov matrix $\Pi(\theta)$. As a consequence, the set $\mathcal{G} \subset \mathbb{R}^{\bar{n}_{\xi}}$ of all controllers satisfying (7) with the Lyapunov matrix $\Pi(\theta)$ turns out to be open, and $\mathcal{G}^{N}$ will be open as well. Finally, when $N \geq 2 M+1$, the set $\mathcal{D}_{N}$ is generic and of full measure on $\mathbb{R}^{N \bar{n}_{\xi}}$ and, hence, $\mathcal{G}^{N} \cap \mathcal{D}_{N}$ is non-negligible.

Proof of Proposition 1: This is a straightforward consequence of the fact that, when $\hat{\theta} \neq \theta$, we cannot have $z(t)=z\left(t, t_{0}, \hat{x}_{0}, \hat{\xi}_{0}, \hat{\theta}, \sigma\right)$ a.e. on $\mathcal{I}$, since the observed closed-loop data are generated as $z(t)=z\left(t, t_{0}, x_{0}, \xi_{0}, \theta, \sigma\right)$ with $\left(x_{0}, \xi_{0}\right) \neq 0$ and the control law $\mathcal{C}_{\sigma}$ is supposed to be discerning. Hence, $\delta_{\sigma}(z, \hat{\theta})>0$ whenever $\hat{\theta} \neq \theta$, and $\delta_{\sigma}(z, \theta)=0$ since by hypothesis $z \in \mathcal{S}_{\sigma}(\theta)$.

Proof of Proposition 2: Recalling that the forced response $z^{(\mathrm{f})}$ of the switching linear system $\left(\mathcal{P}(\theta) / \mathcal{C}_{\sigma}\right)$ can be written 
as

$$
\begin{aligned}
z^{(\mathrm{f})}(t) & =\Lambda_{\sigma(t)}(\theta) \int_{t_{0}}^{t} \Phi_{\sigma}\left(\tau, t_{0}, \theta\right) \Xi_{\sigma(\tau)}(\theta) v(\tau) d \tau \\
& +\Gamma_{\sigma(t)} v(t)
\end{aligned}
$$

it is an easy matter to see that

$$
\begin{aligned}
\left|z^{(\mathrm{f})}(t)\right| & \leq\left|\Lambda_{\sigma(t)}(\theta)\right| \int_{t_{0}}^{t}\left|\Phi_{\sigma}\left(\tau, t_{0}, \theta\right) \Xi_{\sigma(\tau)}(\theta)\right| d \tau\|v\|_{\infty, \mathcal{I}} \\
& +\left|\Gamma_{\sigma(t)}\right||v(t)| .
\end{aligned}
$$

From the latter inequality, the bound in (14) can be readily obtained since, by hypothesis, the switching signal $\sigma$ contains only a finite number of discontinuity points in $\mathcal{I}$.

Proof of Proposition 3: It follows from standard calculations by replacing $z^{(\mathrm{n})}(t)$ with $\Lambda_{\sigma(t)}(\theta) \Phi_{\sigma}\left(t, t_{0}, \theta\right)$ in the expression for the distance $\delta_{\sigma}\left(z^{(\mathrm{n})}, \hat{\theta}\right)$.

Proof of Lemma 3: In view of Proposition 3, we have that

$$
\delta_{\sigma}\left(z^{(\mathrm{n})}, \hat{\theta}\right) \leq \beta_{1}(\theta, \hat{\theta})\left|\chi_{0}\right|
$$

with

$$
\begin{aligned}
& \beta_{1}^{2}(\theta, \hat{\theta})= \\
& \lambda_{\max }\left\{\left[\begin{array}{c}
I \\
-V_{\sigma}(\theta, \hat{\theta})
\end{array}\right]^{\top} W_{\sigma}(\theta, \hat{\theta})\left[\begin{array}{c}
I \\
-V_{\sigma}(\theta, \hat{\theta})
\end{array}\right]\right\} .
\end{aligned}
$$

Notice that $\beta_{1}(\theta, \hat{\theta})$ depends continuously on $\theta$ and $\hat{\theta}$ and, in addition, $\beta_{1}(\theta, \hat{\theta})=0$ if and only if $\theta=\hat{\theta}$ since the switching law is supposed to be discerning. Then, the class $\mathcal{K}$ function $\beta(\rho)$ can be taken equal to $\max _{\theta, \hat{\theta} \in \Theta,|\theta-\hat{\theta}| \leq \rho,} \beta_{1}(\theta, \theta)$. As for the lower bound, notice that Proposition 3 implies also that

$$
\begin{aligned}
{\left[\delta_{\sigma}\left(z^{(\mathrm{n})}, \hat{\theta}\right)\right]^{2} } & \geq \lambda_{\min }\left\{W_{\sigma}(\theta, \hat{\theta})\right\}\left(\left|\chi_{0}\right|^{2}+\left|V_{\sigma}(\theta, \hat{\theta}) \chi_{0}\right|^{2}\right) \\
& \geq \lambda_{\min }\left\{W_{\sigma}(\theta, \hat{\theta})\right\}\left|\chi_{0}\right|^{2} .
\end{aligned}
$$

Since $\lambda_{\min }\left\{W_{\sigma}(\theta, \hat{\theta})\right\}$ depends continuously on $\theta, \hat{\theta}$ and is equal to 0 if and only if $\theta=\hat{\theta}$ (again thanks to the discernibility of the switching law), then a class $K$ function $\alpha(|\theta-\hat{\theta}|)$ can be found that satisfies the inequality $\lambda_{\min }\left\{W_{\sigma}(\theta, \hat{\theta})\right\} \geq$ $\alpha^{2}(|\theta-\hat{\theta}|)$ for any $\theta, \hat{\theta} \in \Theta$. In particular, $\alpha(|\theta-\hat{\theta}|)$ can be constructed as in the proof of Theorem 2 of [21] to which the reader is referred for additional details.

Proof of Theorem 3: Since $\Theta_{L}$ is $\epsilon$-dense in $\Theta$, there exists at least one $\hat{\theta}^{*} \in \Theta_{L}$ such that $\left|\theta-\hat{\theta}^{*}\right| \leq \epsilon$. For such a $\hat{\theta}^{*}$, one has

$$
\begin{aligned}
\delta_{\sigma}\left(z, \hat{\theta}^{*}\right) & \leq \delta_{\sigma}\left(z^{(\mathrm{n})}, \hat{\theta}^{*}\right)+\left\|z^{(\mathrm{f})}\right\|_{2, \mathcal{I}} \\
& \leq \beta\left(\left|\theta-\hat{\theta}^{*}\right|\right)\left|\chi_{0}\right|+\left\|z^{(\mathrm{f})}\right\|_{2, \mathcal{I}} \\
& \leq \beta(\epsilon)\left|\chi_{0}\right|+\left\|z^{(\mathrm{f})}\right\|_{2, \mathcal{I}} .
\end{aligned}
$$

Since the estimate $\hat{\theta}_{L}$ is optimal in $\Theta_{L}$, one has also

$$
\delta_{\sigma}\left(z, \hat{\theta}_{L}\right) \leq \delta_{\sigma}\left(z, \hat{\theta}^{*}\right) \leq \beta(\epsilon)\left|\chi_{0}\right|+\left\|z^{(\mathrm{f})}\right\|_{2, \mathcal{I}} .
$$

Further, by exploiting the lower bound in Proposition 3, we can write

$$
\begin{aligned}
\delta_{\sigma}\left(z, \hat{\theta}_{L}\right) & \geq \delta_{\sigma}\left(z^{(\mathrm{n})}, \hat{\theta}_{L}\right)-\left\|z^{(\mathrm{f})}\right\|_{2, \mathcal{I}} \\
& \geq \alpha\left(\left|\theta-\hat{\theta}_{L}\right|\right)\left|\chi_{0}\right|-\left\|z^{(\mathrm{f})}\right\|_{2, \mathcal{I}} .
\end{aligned}
$$

Combining the two latter inequalities, we obtain

$$
\alpha\left(\left|\theta-\hat{\theta}_{L}\right|\right)\left|\chi_{0}\right| \leq \beta(\epsilon)\left|\chi_{0}\right|+2\left\|z^{(\mathrm{f})}\right\|_{2, \mathcal{I}}
$$

which can be written as 201, Proposition 2 and the fact that any class $\mathcal{K}$ function is invertible.

\section{REFERENCES}

[1] K. Glover and J. Willems, "Parametrizations of linear dynamical systems: Canonical forms and identifiability," IEEE Transactions on Automatic Control, vol. 19, no. 6, pp. 640-646, 1974.

[2] M. Grewal and K. Glover, "Identifiability of linear and nonlinear systems," IEEE Transactions on Automatic Control, vol. 21, no. 6, pp. 833-837, 1976.

[3] M. Blanke, M. Kinnaert, J. Lunze, and M. Staroswiecki, Diagnosis and Fault-Tolerant Control. Springer-Verlag, 2006.

[4] H. Yang, B. Jiang, and V. Cocquempot, Fault Tolerant Control Design for Hybrid Systems. Springer-Verlag, Berlin Heidelberg, 2010.

[5] D.Liberzon, Switching in Systems and Control. Birkhäuser, 2003.

[6] T. Steffen, Control Reconfiguration of Dynamical Systems, ser. Lecture Notes in Control and Information Sciences, M. Thoma and M. Morari, Eds. Berlin Heidelberg: Springer-Verlag, 2005, vol. 320.

[7] K. Åström and B. Wittenmark, Adaptive Control. Addison-Wesley, 1989.

[8] P. Ioannou and J. Sun, Robust Adaptive Control. Prentice Hall, 1996.

[9] G. Battistelli, "On stabilization of switching linear systems," Automatica, vol. 49, pp. 1162-1173, 2013.

[10] M. Baglietto, G. Battistelli, and P. Tesi, "Discerning controllers for switching linear systems: Existence and genericity," Automatica, vol. 50, no. 9, pp. $2358-2365,2014$.

[11] A. Morse and F. Pait, "A cyclic switching strategy for parameter-adaptive contro," vol. 39, no. 6, 1994, pp. 1172-1183.

[12] - "Mimo design models and internal regulators for cyclicly-switched parameter-adaptive control systems," in Proceedings of the 1993 American Control Conference, San Francisco, California, 1993.

[13] A. S. Morse, "Supervisory control of families of linear set-point controllers, part 2: robustness," IEEE Trans. on Automatic Control, vol. 42(11), pp. $1500-1515,1997$.

[14] J. P. Hespanha, D. Liberzon, and A. S. Morse, "Hysteresis-based switching algorithms for supervisory control of uncertain systems," Automatica, vol. 39, pp. 263-272, 2003.

[15] J. Hespanha, D. Liberzon, A. Morse, B. Anderson, T. Brinsmead, and F. de Bruyne, "Multiple model adaptive control, part 2: switching," Int. J. Robust Nonlinear Control, vol. 11(5), pp. 479-496, 2001.

[16] M. Baglietto, G. Battistelli, and P. Tesi, "Stabilization and tracking for switching linear systems under unknown switching sequences," Systems \& Control Letters, vol. 62, pp. 11-21, 2013.

[17] E. Sontag, "For differential equations with $r$ parameters, $2 r+1$ experiments are enough for identification," Journal of Nonlinear Science, vol. 12, no. 6, pp. 553-583, 2003.

[18] B. Anderson, T. Brinsmead, F. de Bruyne, J. Hespanha, D. Liberzon, and A. Morse, "Multiple model adaptive control, part 1: finite controller coverings," Int. J. Robust Nonlinear Control, vol. 10(11), pp. 909-929, 2000.

[19] S. Baldi, G. Battistelli, E. Mosca, and P. Tesi, "Multi-model unfalsified adaptive switching control. test functionals for stability and performance," submitted to International Journal of Adaptive Control and Signal Processing.

[20] D. Buchstaller and M. French, "Robust stability and performance analysis for multiple model adaptive controllers," in Proceedings of the 48th IEEE Conference on Decision and Control and 28th Chinese Control Conference, Shanghai, P.R. China, 2009.

[21] M. Baglietto, G. Battistelli, and P. Tesi, "Distinguishability of discretetime nonlinear systems," IEEE Transactions on Automatic Control, vol. 59, no. 4, pp. 1014-1020, 2014. 